\title{
Cardiac resynchronization therapy and its effects in patients with type 2 DIAbetes mellitus OPTimized in automatic vs. echo guided approach. Data from the DIA-OPTA investigators
}

Celestino Sardu ${ }^{1 *}$ D, Pasquale Paolisso ${ }^{2}$, Valentino Ducceschi ${ }^{3}$, Matteo Santamaria ${ }^{4}$, Cosimo Sacra ${ }^{4}$, Massimo Massetti ${ }^{4,5}$, Antonio Ruocco ${ }^{6}$ and Raffaele Marfella ${ }^{1}$

\begin{abstract}
Objectives: To evaluate the effects of cardiac resynchronization therapy (CRTd) in patients with type 2 diabetes mellitus (T2DM) optimized via automatic vs. echocardiography-guided approach.

Background: The suboptimal atrio-ventricular (AV) and inter-ventricular (VV) delays optimization reduces CRTd response. Therefore, we hypothesized that automatic CRTd optimization might improve clinical outcomes in T2DM patients.

Methods: We designed a prospective, multicenter study to recruit, from October 2016 to June 2019, 191 consecutive failing heart patients with T2DM, and candidate to receive a CRTd. Study outcomes were CRTd responders rate, hospitalizations for heart failure (HF) worsening, cardiac deaths and all cause of deaths in T2DM patients treated with CRTd and randomly optimized via automatic (n 93) vs. echocardiography-guided (n 98) approach at 12 months of follow-up.
\end{abstract}

Results: We had a significant difference in the rate of CRTd responders (68 (73.1\%) vs. 58 (59.2\%), p 0.038), and hospitalizations for HF worsening (12 (16.1\%) vs. 22 (22.4\%), p 0.030) in automatic vs. echocardiography-guided group of patients. At multivariate Cox regression analysis, the automatic guided approach (3.636 [1.271-10.399], Cl 95\%, p 0.016 ) and baseline highest values of atrium pressure (automatic SonR values, 2.863 [1.537-6.231], Cl 95\%, p 0.006) predicted rate of CRTd responders. In automatic group, we had significant difference in SonR values comparing the rate of CRTd responders vs. non responders ( $1.24 \pm 0.72 \mathrm{~g}$ vs. $0.58 \pm 0.46 \mathrm{~g}$ (follow-up), p 0.001), the rate of hospitalizations for HF worsening events $(0.48 \pm 0.29 \mathrm{~g}$ vs. $1.18 \pm 0.43 \mathrm{~g}, \mathrm{p} 0.001)$, and the rate of cardiac deaths $(1.13 \pm 0.72 \mathrm{~g}$ vs. $0.65 \pm 0.69 \mathrm{~g}, \mathrm{p}$ 0.047).

Conclusions: Automatic optimization increased CRTd responders rate, and reduced hospitalizations for HF worsening. Intriguingly, automatic CRTd and highest baseline values of SonR could be predictive of CRTd responders. Notably, there was a significant difference in SonR values for CRTd responders vs. non responders, and about hospitalizations for HF worsening and cardiac deaths.

Clinical trial ClinicalTrials.gov Identifier NCT04547244.

*Correspondence: drsarducele@gmail.com

${ }^{1}$ Department of Advanced Medical and Surgical Sciences, University

of Campania "Luigi Vanvitelli", Piazza Miraglia 2, 80131 Naples, Italy

Full list of author information is available at the end of the article

(c) The Author(s) 2020. This article is licensed under a Creative Commons Attribution 4.0 International License, which permits use, sharing, adaptation, distribution and reproduction in any medium or format, as long as you give appropriate credit to the original author(s) and the source, provide a link to the Creative Commons licence, and indicate if changes were made. The images or other third party material in this article are included in the article's Creative Commons licence, unless indicated otherwise in a credit line to the material. If material is not included in the article's Creative Commons licence and your intended use is not permitted by statutory regulation or exceeds the permitted use, you will need to obtain permission directly from the copyright holder. To view a copy of this licence, visit http://creativeco mmons.org/licenses/by/4.0/. The Creative Commons Public Domain Dedication waiver (http://creativecommons.org/publicdomain/ zero/1.0/) applies to the data made available in this article, unless otherwise stated in a credit line to the data. 
Keywords: Type 2 diabetes mellitus, Cardiac resynchronization therapy, Automatic CRTd optimization

\section{Background}

Type 2 diabetes mellitus (T2DM) is a risk factor, that negatively impacts on clinical prognosis for patients with heart failure (HF), and in those receiving a Cardiac resynchronization therapy with defibrillator (CRTd), [1]. On other hand, the CRTd could ameliorate clinical outcomes, because it has a positive impact on both morbidity and mortality in treated patients [2]. Notably, the T2DM accounts about the $38 \%$ of patients treated with a CRTd [1], and the patients which do not respond to CRTd are defined "CRTd non responders", and are those with worse prognosis [3]. In this setting, the T2DM is a leading cause of multiple and complex alterations of molecular, metabolic, electrical, and mechanical cardiac functions, which cause arrhythmias and worsening of cardiac pump [1]. Consequently, the worsening of cardiac pump is a relevant cause of hospitalizations and deaths in CRTd patients [1-5]. Thus, in last decades a great effort has been invested to develop new therapeutic approaches to improve the cardiac pump efficiency, the number of CRTd responders and the clinical outcomes in CRTd patients with T2DM. In this setting, the use of multipolar left-ventricular (LV) pacing leads, and the optimization of CRTd device programming mode has been seen as an important advancement in T2DM patients with CRTd $[6,7,8]$. On other hand, also T2DM patients receiving a multipolar CRTd could experience a worse prognosis [6]. This could be caused by the reduction of cardiac pump, which is more evidenced in patients with the loss of atrio-ventricular (AV) and inter-ventricular (IV) synchrony [9]. Therefore, the optimization of AV and IV intervals could be a therapeutic target, to ameliorate the CRTd effectiveness, and to increase the rate of CRTd responders [8, 9]. By the way, the echocardiography could be used to ameliorate the optimization of AV/IV intervals [9]. On other hand, echocardiography showed contrasting results in clinical studies, and low application in clinical practice [9]. Therefore, new techniques, as the intracardiac electrogram (IEGM) guided approach, have been proposed for the optimization of AV/IV intervals in CRTd patients [9]. Indeed, the IEGM-guided approach is faster, simpler, and it is a reliable alternative to the echo-guided approach for CRTd optimization [9]. On the other hand, the IEGM-guided approach showed contrasting results in the optimization of the CRTd [9]. Furthermore, authors showed its inferiority as compared to echocardiography-guided approach about the hemodynamic outcome [9]. Therefore, recently authors have introduced a new optimization technique, that is not IEGM-guided [8]. This new optimization technique is correlated with $\mathrm{dP} / \mathrm{dt}$ max of $\mathrm{LV}$, and with the hemodynamic function of the heart [8]. To date, this non IEGM-guided technique evaluates the peak of endocardial acceleration during isovolumetric contraction of the left ventricle, and its amplitude, that is recorded as SonR signal $[8,10]$. Therefore, the values of SonR are related to the contractile function of the heart [8], and the automatic vs. echo-guided approach could lead to an increase of response to the CRTd [10]. However, our study hypothesis was that automatic vs. echo-guided approach might result in best optimization of AV/IV delays in T2DM patients treated by multipolar CRTd. In addition, changes in SonR signals could be seen in diabetic patients with CRTd who experienced the main clinical outcomes. Thus, automatic vs. echo-guided CRTd optimization could result in reduction of CRTd responders, of hospitalizations for HF worsening and deaths (cardiac deaths and all causes of death) in HF patients with T2DM treated with multipolar CRTd. Therefore, in the present study we assessed the rate of CRTd responders, the hospitalizations for HF worsening and the deaths (cardiac deaths and all causes of death) in HF patients with T2DM treated with multipolar CRTd, and randomly assigned to the automatic vs. echo-guided group of CRTd optimization at 12 months of follow-up. Finally, we assessed the SonR values at baseline and at follow-up of 12 months for CRTd responders, for patients with hospitalizations for HF worsening, and for deaths events.

\section{Methods \\ Study design}

Between 11th January 2010 and 20th January 2019, we screened a population of 203 consecutive patients with T2DM, chronic HF and indication to receive a CRTd in an observational multicenter, randomized study (DIAOPTA investigators) Fig. 1. The diagnosis of T2DM was made according to American Diabetes Association criteria [11]. To establish T2DM patients treatment, the screened patients answered a specific questionnaire about medicines used for diabetes treatment, with the date of the beginning and end of treatment, route of administration, and duration of use [11]. The diagnosis of HF was made as indicated by international guidelines on HF disease management [12]. Moreover, only patients with T2DM and HF were enrolled in the study, according to inclusion/exclusion criteria. Figure 1. The study 


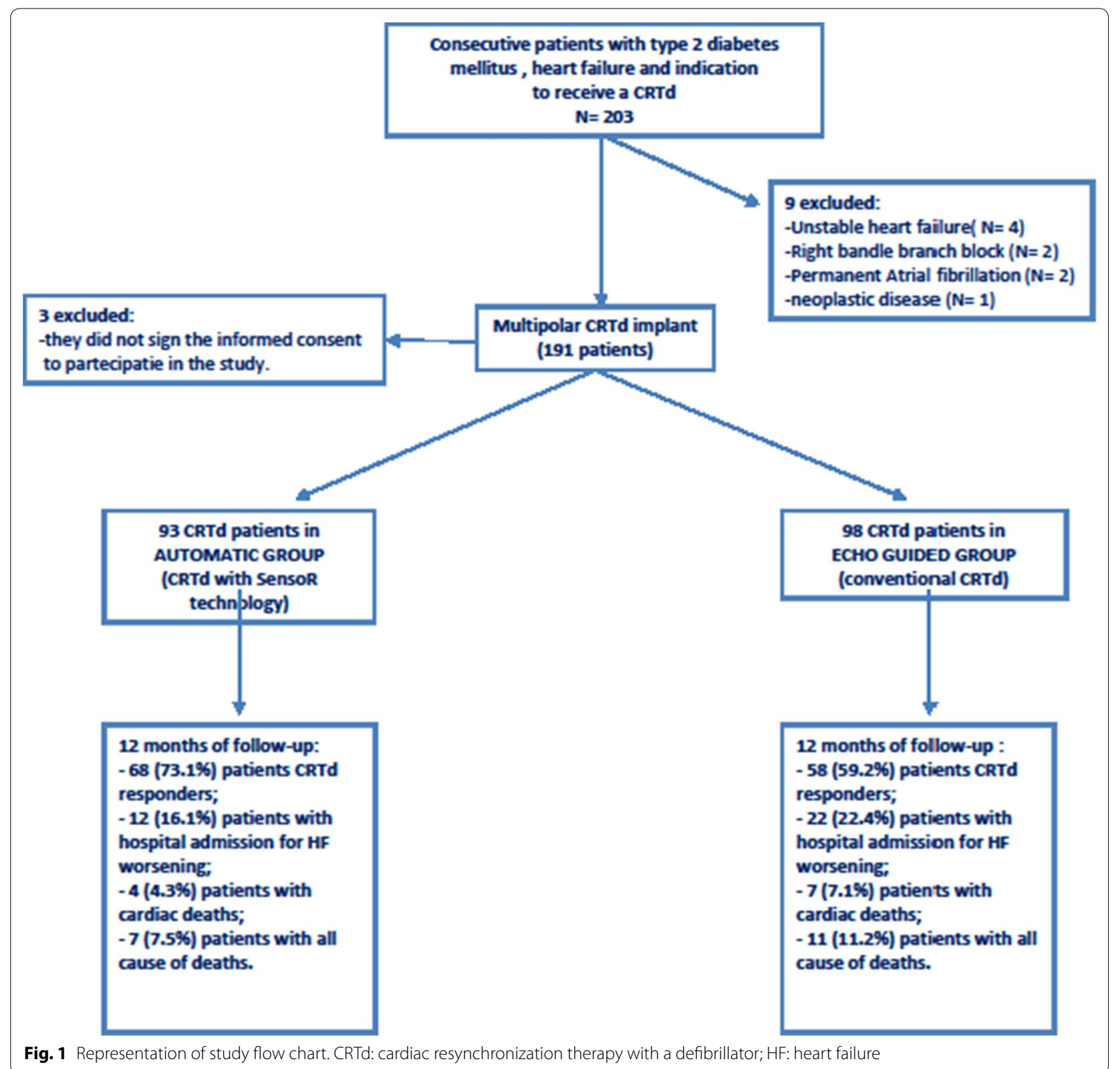

population respected the following inclusion/exclusion criteria:

Inclusion criteria: at least 18 years of age, T2DM diagnosis, with clinical history of stable chronic heart failure, New York Heart Association (NYHA) functional class II or III, sinus rhythm, left bundle branch block, severe left ventricle ejection fraction reduction ( $\mathrm{LVEF}<35 \%)$, stable sinus rhythm, and candidates to receive a CRT-d treatment [12].

Exclusion criteria: age $<18$ or $>75$ years, ejection fraction $>35 \%$, previous implant of implantable cardioverter defibrillator (ICD), CRT-d and/or pacemaker, absence of informed patient consent, and any condition that would make survival for 1 year unlikely.

\section{Study population and intervention}

The 191 enrolled patients with T2DM and HF respected the clinical indication for implantation of a de-novo multipolar CRTd, according to current international guidelines [12].

Thus, we randomly treated the patients with T2DM via conventional CRTd implant (n 98) vs. SensoR-CRTd 
(n 93), using a computer generating a code program. The patients with conventional CRTd implant were optimized using echo-guided approach, and defined as "Echo group". The patients with automatic sensor guided CRTd were defined as "Automatic group", and they were not optimized by echo-guided approach Fig. 1. However, in a time of 14 days after a successful CRTd implant, patients were randomized (2:1, respectively) to weekly automatic AV and IV delay optimization with SonR in Automatic group vs. echo-guided optimization in Echo group. The full description of CRTd implant (Automatic vs. Echo group) is provided in Additional file 1.

At baseline and for all follow-up duration (6 and 12 months) the patients underwent full echocardiographic evaluation, and a global clinical status (NYHA) assessment, and CRTd device interrogation [13]. Before CRTd intervention and during follow-up, we determined the baseline laboratory studies by peripheral blood and enzymatic assays after an overnight fast (values of plasma glucose, glycated hemoglobin 1Ac type (HbA1c), B type natriuretic peptide (BNP) and serum lipids). In addition, at baseline, and during follow up we measured inflammatory markers as circulating serum levels of proinflammatory cytokines (tumor necrosis factor- $\alpha$, TNF $\alpha$, interleukin-6, IL6), systemic inflammatory markers (C reactive protein, CRP), and leucocytes and neutrophils count as previously reported [13].

Thereafter, for each enrolled patient during clinical, instrumental assessment, and device telemetric control (at implant, 10 days, 6, and 12 months after discharge) and by visualization of hospital discharge schedules, we reported the effects of CRT-d in terms of clinical outcomes, CRT responders rate, and clinical events as hospitalizations for HF worsening, and deaths. The full description is reported in Additional file 1.

\section{Echocardiographic evaluation}

Two experienced physicians in echocardiography (Co. S, M.M), performed at baseline, and at 6th and 12th month of follow up, a trans-thoracic two-dimensional echocardiogram with M-mode, conventional Doppler, and pulsedwave tissue Doppler imaging (TDI) measurements in each patient using a Philips iE33 echocardiograph (Eindhoven, The Netherlands). The images of echocardiography were acquired in the parasternal long and short axis views. However, we calculated LV end-diastolic diameter (LVEDD), end-diastolic volume (LVEDV), end-systolic diameter (LVESD), end-systolic volume (LVESV), and then we determined LV ejection fraction (LVEF) with the Simpson biplane method [14]. In addition, the amount of mitral regurgitation was calculated as the area of the color-flow Doppler regurgitant jet divided by the area of the left atrium in systole, and described as low (+), moderate $(++)$, moderate-severe $(+++)$, and severe $(++++),[14,15]$. To have final calculation measures the physicians performed systematically averaged measurements in five consecutive samples. The physicians involved in echocardiographic baseline and follow-up evaluation, performed and analyzed each exam in independent way, and blinded to the study protocol. In a time of 14 days after a successful CRTd implant, patients were randomized (2:1, respectively) to the automatic atrioventricular (AV) and inter-ventricular (IV) delay optimization with SonR (Automatic group) vs. Echo-guided optimization (Echo group), using a mandatory standardized protocol [16, 17]. At baseline and at follow-up, we performed the echocardiography measurements in CRTd patients at rest, and in supine position [12]. Finally, all measurements were reviewed by two observers blinded to measures performed previously by other observers. In addition, the observers were blinded to study protocol and to the group of study that the patient had been selected for.

\section{Evaluation of SonR signals and automatic optimization of AV/IV intervals}

After CRTd implant the patients in Automatic and Echo group were evaluated by device interrogation at followup, as previously described by authors [10]. Specifically, for patients in the automatic group, during CRTd interrogation, we evaluated the modifications (baseline vs. follow-up values) of signals recorded by SonR sensor, that is allocated in right atrium lead [10]. Intriguingly, the values of SonR signals are recordings of endocardial acceleration signals of the heart, that correspond to the mechanical vibrations of myocardium during cardiac contraction $[8,10]$. Thus, during the isovolumetric contraction phase of the cardiac cycle we registered the highest amplitude of the SonR signal, that correspond to the cardiac contractility [10]. However, there is a correlation between the amplitude of the recorded SonR signal and LV dP/ dtmax, and so it could be seen as index of the contractile function of the heart [10]. Indeed, the amplitude of SonR signal corresponds to the first heart sound, and it is a surrogate index of systolic function of heart [10]. Furthermore, from registration and evaluation of SonR signals, the CRT-d automatically adjusts the AV/IV delays, on a weekly basis, at rest and during exercise in the automatic group [10]. Finally, a concordance has been showed between echocardiographic methods and SonR device based method used for AV/IV delays optimization [10].

\section{Echocardiographic optimization of the atrioventricular and interventricular intervals}

In the echo-guided group, we optimized the AV and IV intervals via echocardiography during continuous 
Table 1 Clinical characteristics of study population at baseline in overall, and automatic vs. echo-guided patients

\begin{tabular}{|c|c|c|c|c|}
\hline Parameters & Overall population (n 191) & Automatic (n 93) & Echo guided (n 98) & P value \\
\hline Age & $71 \pm 6$ & $71 \pm 7$ & $72 \pm 6$ & 0.426 \\
\hline Male (\%) & $134(70.2)$ & $63(67.7)$ & $71(72.4)$ & 0.431 \\
\hline Smokers (\%) & $97(50.8)$ & $46(49.5)$ & $51(52)$ & 0.407 \\
\hline Hypertension (\%) & $136(71.2)$ & 65 (69.9) & $71(72.5)$ & 0.282 \\
\hline Dislipidemia (\%) & $71(37.2)$ & $35(37.6)$ & $36(36.7)$ & 0.462 \\
\hline Plasma glucose (mg/dl) & $186.7 \pm 22.1$ & $185.5 \pm 22.3$ & $188.9 \pm 22.0$ & 0.367 \\
\hline $\mathrm{HbA} 1 \mathrm{c}(\mathrm{mmol} / \mathrm{mol})$ & $57.9 \pm 16.3$ & $57.8 \pm 16.2$ & $58.1 \pm 16.4$ & 0.263 \\
\hline $\mathrm{BMI}>30 \mathrm{~kg} / \mathrm{m}^{2}(\%)$ & $15(7.8)$ & $8(8.6)$ & $7(7.1)$ & 0.791 \\
\hline COPD (\%) & $35(18.3)$ & $17(18.3)$ & $18(18.4)$ & 0.538 \\
\hline Renal disease (\%) & $35(18.3)$ & $16(17.2)$ & $19(19.4)$ & 0.105 \\
\hline Ischemic heartfailure (\%) & $131(68.6 \%)$ & $65(69.9)$ & $66(67.4)$ & 0.302 \\
\hline II NYHA class (\%) & $33(25.2)$ & $16(24.6)$ & $17(25.8)$ & 0.280 \\
\hline III NYHA class (\%) & $98(74.8)$ & $49(75.4)$ & $49(74.2)$ & 0.211 \\
\hline QRS duration (ms) & $137.4 \pm 9.2$ & $137.5 \pm 9.0$ & $137.9 \pm 9.4$ & 0.930 \\
\hline $6 \mathrm{MWT}$ & $243.47 \pm 41.83$ & $241.18 \pm 44.94$ & $246.75 \pm 40.74$ & 0.371 \\
\hline SonR values (g) & / & $0.24 \pm 0.08$ & / & / \\
\hline \multicolumn{5}{|c|}{ Echocardiographic parameters } \\
\hline LVEF (\%) & $27 \pm 8$ & $27 \pm 5$ & $28 \pm 5$ & 0.285 \\
\hline LVEDd (mm) & $65 \pm 8$ & $66 \pm 7$ & $64 \pm 9$ & 0.101 \\
\hline LVESd (mm) & $43 \pm 8$ & $41 \pm 6$ & $44 \pm 9$ & 0.291 \\
\hline LVEDV (ml) & $205 \pm 20$ & $206 \pm 18$ & $203 \pm 22$ & 0.993 \\
\hline LVESV (ml) & $146 \pm 17$ & $148 \pm 15$ & $145 \pm 18$ & 0.818 \\
\hline \multicolumn{5}{|l|}{ Mitral insufficiency } \\
\hline$+(\%)$ & $96(50.3)$ & $45(48.4)$ & $51(52.0)$ & 0.359 \\
\hline$++(\%)$ & $78(40.8)$ & $38(40.9)$ & $40(40.8)$ & 0.556 \\
\hline$+++(\%)$ & $17(8.9)$ & $10(10.7)$ & $7(7.2)$ & 0.451 \\
\hline \multicolumn{5}{|l|}{ Medications at baseline } \\
\hline Amiodarone (\%) & $40(20.9)$ & $19(20.4)$ & $21(21.4)$ & 0.569 \\
\hline ACE inhibitors (\%) & $86(45)$ & $42(45.2)$ & $44(44.9)$ & 0.543 \\
\hline ARS blockers (\%) & $61(31.9)$ & $31(33.3)$ & $30(30.6)$ & 0.464 \\
\hline Sacubitril/valsartan (\%) & $47(24.6)$ & $23(24.7)$ & $24(24.5)$ & 0.551 \\
\hline \multicolumn{5}{|l|}{ Beta blockers: } \\
\hline Carvedilol (\%) & $74(38.7)$ & $36(38.7)$ & $38(38.8)$ & 0.555 \\
\hline Bisoprolol (\%) & $62(32.5)$ & $32(34.4)$ & $30(30.6)$ & 0.539 \\
\hline Aspirin (\%) & 76 (39.8) & $36(38.7)$ & $40(40.8)$ & 0.558 \\
\hline Tiklopidine(\%) & $5(2.6)$ & $2(2.1)$ & $3(3.1)$ & 0.525 \\
\hline Warfarin (\%) & $57(29.8)$ & $27(29)$ & $30(30.6)$ & 0.468 \\
\hline NOAC (\%) & 45 (23.6) & $20(21.5)$ & $25(25.5)$ & 0.316 \\
\hline Calcium antagonist (\%) & $12(6.3)$ & $5(5.4)$ & $7(7.1)$ & 0.501 \\
\hline Ivabradine(\%) & $40(20.9)$ & $21(22.6)$ & 19 (19.4) & 0.599 \\
\hline Digoxin (\%) & $57(29.8)$ & $27(29)$ & $30(30.6)$ & 0.468 \\
\hline Loop diuretics (\%) & $168(88)$ & $79(84.9)$ & $89(90.8)$ & 0.268 \\
\hline Aldosterone Blockers (\%) & $117(61.3)$ & $55(59.1)$ & $62(63.3)$ & 0.656 \\
\hline Statins (\%) & $142(74.3)$ & $69(74.2)$ & $73(74.5)$ & 0.461 \\
\hline \multicolumn{5}{|l|}{ Anti diabetic drugs, n (\%) } \\
\hline Insulin (\%) & $40(20.9)$ & $18(19.3)$ & $22(22.4)$ & 0.722 \\
\hline Metformin (\%) & $109(57.1)$ & $49(52.7)$ & $60(61.2)$ & 0.246 \\
\hline Sulfonylureas (\%) & $34(17.8)$ & $16(17.2)$ & $18(18.4)$ & 0.852 \\
\hline Thiazolidinediones (\%) & $22(11.5)$ & $10(10.7)$ & $12(12.2)$ & 0.823 \\
\hline
\end{tabular}


Table 1 (continued)

\begin{tabular}{lllll}
\hline Parameters & Overall population $(\mathbf{n}$ 191) & Automatic $(\mathbf{n}$ 93) & Echo guided (n 98) & P value \\
\hline GLP-1 agonist (\%) & $28(14.7)$ & $13(14)$ & $15(15.3)$ & $22(22.5)$ \\
DPP-4 inhibitors (\%): & $40(20.9)$ & $18(19.3)$ & & \\
Biomarkers & & & $7.840 \pm 2.35$ & 0.722 \\
Lymphocytes & $7.95 \pm 2.29$ & $7.99 \pm 2.23$ & $5.37 \pm 1.90$ & 0.271 \\
Neutrophiles & $5.38 \pm 1.92$ & $5.40 \pm 1.95$ & $332.74 \pm 19.55$ & 0.421 \\
BNP (pg/ml) & $327.38 \pm 18.61$ & $321.04 \pm 18.72$ & $9.36 \pm 0.97$ & 0.667 \\
CRP (mg/L) & $9.84 \pm 0.94$ & $10.36 \pm 1.03$ & $6.47 \pm 0.06$ & 0.466 \\
IL6 (pg/ml) & $6.42 \pm 0.05$ & $6.38 \pm 0.04$ & $6.29 \pm 0.02$ & 0.272 \\
TNFa (pg/ml) & $6.31 \pm 0.03$ & $6.34 \pm 0.03$ & 0.269 \\
\hline
\end{tabular}

ACE, Angiotensin Converting Enzyme; ARS, Angiotensin Receptors; BMI, body mass index; BNP, B type natriuretic peptide; COPD, chronic obstructive pulmonary disease; CRP, C reactive protein; DPP-4, dipeptidyl peptidase-4; HbA1c, glycated hemoglobin 1Ac type; GLP-1, glucagone like peptide-1; IL-6, interleukine 6; LVEDd, left ventricle end diastolic diameter; LVEDv, left ventricle end diastolic volume; LVEF, left ventricle ejection fraction; LVESd, left ventricle end systolic diameter; LVESv, left ventricle end systolic volume; NYHA II, III, New York Heart Association II and III class; NOAC, new oral anti coagulation; SonR, values of SonR signals; TNFa, tumor necrosis factor alpha; 6MWT, 6 min walking test.* is for statistical significant $(p<0.05)$

ECG monitoring in each patient, and guided by a careful analysis of the 12 -lead ECG [16]. For AV interval optimization we followed the recommendations of the American Society of Echocardiography, via the simplified pulsed Doppler mitral inflow technique [16]. Thus, the AV delay optimization was performed by the evaluation of trans mitral flow using the pulsed wave Doppler [16, 17]. Moreover, we then optimized the LV diastolic filling so that mitral valve closure coincided with the end of the Doppler A wave during ventricular systole [17]. However, it was integrated with the optimized AV delay by the aortic velocity-time integral (VTI) method, by assessing the VTI of flow across the aortic valve [17]. Indeed, VTI measures are directly proportional to LV stroke volume $[16,17]$. Thus, we programmed the VV interval as AV delay optimization by using the aortic VTI method [17]. However, after the determination of the optimal AV delay programming, we performed the VV interval optimization to decrease LV dyssynchrony, by providing a more simultaneous LV activation and reducing the mitral regurgitation in some patients [17].

\section{Study endpoints}

Primary endpoints were the rate of CRTd responders comparing patients in Automatic vs. Echo group. Secondary study endpoints were the hospitalizations for HF worsening, cardiac deaths and all cause of deaths events comparing patients in Automatic vs. Echo group. In addition, in Automatic group of patients we evaluated the amplitude of SonR signals at baseline, and their variations at follow-up for the CRTd responders vs. non responders, and for the events of hospitalization for HF worsening, cardiac deaths and all cause of deaths.

\section{Definition of CRTd responders}

CRT responders were defined, according to authors, by evidence of clinical and echocardiographic diagnostic criteria [12]. Thus, clinically the CRTd responders showed the improvement in NYHA functional class (at least one class) and the increase of the 6 min walk distance $>10 \%$, [12]. At echocardiography, the CRTd responders showed a reduction $\mathrm{LVESD}>15 \%$, and an improvement in LVEF $>10 \%$, [12]. In addition to clinical and instrumental evaluation, authors identified CRTd responders patients also by chest X-rays, to assess reduction in cardiac size and pulmonary congestion [12].

The primary and secondary study endpoints were evaluated at follow-up of 12 months during visits and controls, and by hospital discharge schedules. The detailed description of secondary study endpoint diagnostic criteria, and of study endpoints data collection and analysis was reported in Additional file 1.

\section{Ethical Committee and Clinical trial registration}

Authors conducted the study in accordance with the Declaration of Helsinki. The Ethics Committees of all participating institutions approved the protocol. All patients were informed about the study nature, and gave their written informed, and signed consent to participate in the study. The study was registered in ClinicalTrials.gov, clinical trial number NCT04547244. The authors and investigators of DIA-OPTA study accepted 
Table 2 Clinical characteristics of study population at 12th month of follow-up in overall, and automatic vs. echoguided patients

\begin{tabular}{|c|c|c|c|}
\hline Parameters & Automatic (n 93) & Echo guided (n 98) & $P$ value \\
\hline \multicolumn{4}{|l|}{12 months follow up } \\
\hline $\mathrm{BMI}>30 \mathrm{~kg} / \mathrm{m}^{2}(\%)$ & $7(7.5)$ & $6(6.1)$ & 0.622 \\
\hline $\begin{array}{l}\text { Plasma glucose } \\
(\mathrm{mg} / \mathrm{dl})\end{array}$ & $173.5 \pm 21.7$ & $171.2 \pm 20.9$ & 0.171 \\
\hline $\mathrm{HbA1c}(\mathrm{mmol} / \mathrm{mol})$ & $52.9 \pm 12.1$ & $52.3 \pm 12.0$ & 0.122 \\
\hline I NYHA class & $6(6.4)$ & $2(2.0)$ & $0.016^{*}$ \\
\hline II NYHA class & $45(48.4)$ & $21(21.4)$ & $0.010^{*}$ \\
\hline III NYHA class & $38(40.9)$ & $66(67.3)$ & $0.001^{*}$ \\
\hline IV NYHA class & $4(4.3)$ & $10(10.2)$ & $0.021^{*}$ \\
\hline QRS duration & $121.6 \pm 9.6$ & $122.9 \pm 9.1$ & 0.251 \\
\hline $6 \mathrm{MWT}$ & $319.37 \pm 26.92$ & $227.92 \pm 28.19$ & $0.005^{*}$ \\
\hline SonR values (g) & $1.09 \pm 0.07$ & / & / \\
\hline \multicolumn{4}{|c|}{ Echocardiographic parameters } \\
\hline LVEF (\%) & $36 \pm 6$ & $27 \pm 5$ & $0.001 *$ \\
\hline LVEDd (mm) & $63 \pm 5$ & $65 \pm 8$ & 0.051 \\
\hline LVESd (mm) & $35 \pm 4$ & $38 \pm 5$ & $0.001 *$ \\
\hline LVEDv (ml) & $165 \pm 24$ & $178 \pm 41$ & 0.054 \\
\hline LVESV (ml) & $109 \pm 12$ & $126 \pm 18$ & $0.001^{*}$ \\
\hline \multicolumn{4}{|l|}{ Mitral insufficiency } \\
\hline$+(\%)$ & $50(53.8)$ & $31(31.6)$ & $0.040^{*}$ \\
\hline$++(\%)$ & $38(40.9)$ & $57(58.2)$ & $0.004^{*}$ \\
\hline$+++(\%)$ & $5(5.4)$ & $10(10.2)$ & 0.285 \\
\hline \multicolumn{4}{|l|}{ Biomarkers } \\
\hline Lymphocytes & $7.12 \pm 1.27$ & $8.48 \pm 1.18$ & $0.001 *$ \\
\hline Neutrophiles & $4.87 \pm 1.85$ & $5.69 \pm 2.31$ & $0.001^{*}$ \\
\hline BNP (pg/ml) & $148.41 \pm 16.40$ & $197.26 \pm 19.12$ & $0.001^{*}$ \\
\hline CRP (mg/L) & $7.24 \pm 0.56$ & $8.69 \pm 0.83$ & $0.036^{*}$ \\
\hline IL6 (pg/ml) & $5.55 \pm 0.03$ & $6.31 \pm 0.03$ & $0.011^{*}$ \\
\hline TNFa (pg/ml) & $5.35 \pm 0.02$ & $6.31 \pm 0.02$ & $0.005^{*}$ \\
\hline \multicolumn{4}{|l|}{ Study outcomes } \\
\hline $\begin{array}{l}\text { CRTd responders } \\
(\%)\end{array}$ & $68(73.1)$ & $58(59.2)$ & $0.038^{*}$ \\
\hline $\begin{array}{l}\text { Hospital admission } \\
\text { for HF worsen- } \\
\text { ing (\%) }\end{array}$ & $12(16.1)$ & $22(22.4)$ & $0.030^{*}$ \\
\hline Cardiac deaths (\%) & $4(4.3)$ & $7(7.1)$ & 0.538 \\
\hline $\begin{array}{l}\text { All cause of deaths } \\
(\%)\end{array}$ & $7(7.5)$ & $11(11.2)$ & 0.461 \\
\hline
\end{tabular}

$\mathrm{BMI}$, body mass index; BNP, B type natriuretic peptide; CRP, C reactive protein; HbA1c, glycated hemoglobin 1Ac type; IL-6, interleukine 6; LVEDd, left ventricle end diastolic diameter; LVEDV, left ventricle end diastolic volume; LVEF, left ventricle ejection fraction; LVESd, left ventricle end systolic diameter; LVESv, left ventricle end systolic volume; NYHA II, III, New York Heart Association II and III class; SonR, values of SonR signals; TNFa, tumor necrosis factor alpha; 6MWT, 6 min walking test.** is for statistical significant $(p<0.05)$

full responsibility for the accuracy and completeness of the data and all analyses, and for the fidelity of this report of the trial protocol.

\section{Statistical analysis}

The collected data were analyzed by a qualified statistician. The T2DM patients with CRTd were divided into automatic vs. echo group of patients (conventional group or controls), and during follow up visits, and controls in CRT-d responders vs. CRT-d non-responders. Moreover, we supposed that the number of patients with alterations in primary and secondary endpoints was significantly different between the two groups of patients. Safety analyzes were performed on data from all enrolled patients. Thus, we expressed the continuous variables as means and standard deviations, that were tested by two-tailed Student $\mathrm{t}$ test for paired or unpaired data, as appropriate, or by one-way analysis of variance (ANOVA) for more than two independent groups of data. The categorical variables were compared by Chi square or Fisher exact test where appropriate. We performed survival analysis by the Kaplan Meier method, and we evaluated the predictors of the study endpoints by Cox regression models in patients with automatic as compared with echo-guided CRTd. However, we conducted an univariate analysis to examine the association between single principal clinic, echocardiographic, electrocardiographic characteristics, etc. and automatic CRTd effects, and 12 months study outcomes (CRTd responders rate, hospitalizations for HF worsening, all cause of deaths and cardiac deaths). However, Cox models were adjusted for; age, Body mass index, cholesterol, dyslipidemia, beta-blockers, ace-inhibitors, calcium inhibitors, etc. Therefore, only variables presenting a $\mathrm{p}$ value $\leq 0.25$ at the univariate analysis were included in the model. We used a stepwise method with backward elimination. and we calculated odds ratios (OR) with 95\% confidence intervals. The model was evaluated with Hosmer and Lemeshow test. A 2-sided $\mathrm{p}<0.05$ was considered statistically significant. The statistical analysis was performed using the SPSS software package for Windows 17.0 (SPSS Inc., Chicago Illinois).

\section{Results}

In the present study we analyzed 191 T2DM patients with multipolar CRTd, divided in Automatic group (n 93), vs. Echo group (conventional CRTd implant, n 98) Fig. 1. Characteristics of study population at baseline were reported in Table 1.

At 12th month of follow up, patients in automatic vs. echo group showed a significant reduction of NYHA class, BNP values $(148.41 \pm 16.40$ vs. $197.26 \pm 19.12 \mathrm{pg} /$ $\mathrm{ml}, \mathrm{p} \mathrm{0.001)}$, and inflammatory markers values, with higher values of $6 \mathrm{MWT}(319.37 \pm 26.92$ vs. 
(See figure on next page.)

Fig. 2 a In upper part the SonR values (g) at baseline (left part) and at follow-up end in CRTd responders (green color) vs. CRTd non responders (red color) with the corresponding $\mathrm{p}$ value. In lower part the SonR values in $\mathrm{g}$ at baseline (left part) and at follow-up end in patients with hospital admission for heart failure (HF) worsening (red color) vs. patients without hospital admission for heart failure (HF) worsening (green color) with the corresponding $p$ value. ${ }^{*}$ is for statistical significant $(p<0.05)$. $\mathbf{b}$ In upper part the SonR values in $g$ at baseline (left part) and at follow-up end for patients with all cause of deaths (red color) vs. survived patients (green color) with the corresponding $p$ value. In lower part the SonR values in $g$ at baseline (left part) and at follow-up end in patients with cardiac deaths (red color) vs. survived patients (green color) with the corresponding $p$ value. ${ }^{*}$ is for statistical significant $(p<0.05)$

$227.92 \pm 28.19)$, significant reduction of left ventricle systolic diameters/volumes and mitral valve insufficiency $(\mathrm{p}<0.05)$, and significant improvement of LVEF (36 \pm 6 vs. $27 \pm 5$, p 0.001$)$ Table 2.

As primary study endpoints, comparing patients in automatic vs. echo group, we had a significant higher rate of CRTd responders (68 (73.1\%) vs. 58 (59.2\%), p value 0.038 ) at 12 months of follow-up Table 2.

As secondary study endpoints, comparing patients in automatic vs. echo group, we had a significant lower rate of hospitalizations for HF worsening (12 (16.1\%) vs. $22(22.4 \%), p$ value 0.030$)$ at 12 months of follow-up Table 2.

Intriguingly, at baseline in the automatic group of CRTd patients we did not find a significant difference in SonR values comparing CRTd responders vs. non responders $(0.27 \pm 0.07 \mathrm{~g}$ vs. $0.195 \pm 0.05 \mathrm{~g}$, p 0.055), the patients with vs. those without hospital admissions for HF worsening $(0.25 \pm 0.08 \mathrm{~g}$ vs. $0.24 \pm 0.08 \mathrm{~g}$, p 0.468), the patients with vs. those without all cause of deaths $(0.26 \pm 0.05 \mathrm{~g}$ vs. $0.24 \pm 0.08 \mathrm{~g}, \mathrm{p} 0.642)$ and the patients with vs. those without cardiac deaths $(0.27 \pm 0.04$ g vs. $0.24 \pm 0.08 \mathrm{~g}, \mathrm{p} 0.358)$. Figure 2 . At follow-up end, this trend was confirmed only for all cause of deaths $(0.81 \pm 0.19 \mathrm{~g}$ vs. $1.10 \pm 0.08 \mathrm{~g}, \mathrm{p} 0.437)$, while there was a statistical significant difference about SonR values comparing CRTd responders vs. non responders ( $1.24 \pm 0.72 \mathrm{~g}$ vs. $0.58 \pm 0.46 \mathrm{~g}$ (follow-up), p 0.001), hospital admissions for HF worsening events $(0.48 \pm 0.29 \mathrm{~g}$ vs. $1.18 \pm 0.43 \mathrm{~g}, \mathrm{p} 0.001)$, and cardiac deaths ( $1.13 \pm 0.72$ g vs. $0.65 \pm 0.69$ g, p 0.047) Fig. 2 .

At multivariate Cox regression analysis, automatic CRTd (HR 3.636, [1.271-10.399] CI 95\%, p 0.016), and baseline SonR values (HR 2.863, [1.537-6.231] CI $95 \%, \mathrm{p} 0.006$ ) were predictors of CRTD responders rate Tables 3.

Finally, the Kaplan curves showed the cumulative survival free from CRTd non responders, from hospitalization for HF worsening, from cardiac deaths and all cause of deaths in automatic vs. echo group of CRTd patients Fig. 3.

\section{Discussion}

In the present study, we investigated the effects of automatic vs. echo-guided CRTd optimization in patients with T2DM. Thus, in patients with T2DM we reported the ameliorative effects of automatic vs. echo-guided CRTd optimization approach in terms of significant increase of CRTd responders, and of significant reduction of hospital admissions for HF worsening at follow-up end of 12 months $(p<0.05)$. Notably, for first time in literature we investigated at baseline (CRTd implant) and for all follow-up the values of SonR in the Automatic group of T2DM patients with CRTd. Thus, there were significant modifications of SonR values in CRTd responders vs. non responders patients, and for hospital admissions for HF worsening and for events of cardiac deaths. Finally, and clinically relevant, for T2DM patients the choice of SonR guided automatic CRTd implant could predict a 3.6 folds higher possibility to be CRTd responder. In addition, the patients with higher values of SonR at baseline could have a 2.8 folds higher possibility to become CRTd responders.

Indeed, the automatic lead sensor of right atrium could assess the peak of highest values of atrium pressure [8]. The peak of atrium pressure, as indicated by SonR values, is linked to $\mathrm{LV} \mathrm{dP/dt} \mathrm{max} \mathrm{at} \mathrm{baseline,} \mathrm{and} \mathrm{to} \mathrm{the} \mathrm{endo-}$ cardial acceleration during LV isovolumetric contraction $[8,10]$. Therefore, the amplitude of SonR values is correlated with the heart hemodynamic function, and specifically with the cardiac contractile function $[8,10]$. Therefore, we could speculate that modifications of cardiac contractility correspond to modifications of $\mathrm{dP} / \mathrm{dT}$ values, and to modifications of SonR signals. Furthermore, in HF patients with T2DM the automatic vs. echoguided CRTd optimization could significantly reduce the levels of inflammatory biomarkers (CRP, IL6, TNFa), and of BNP values via its favorable hemodynamic and clinical effects. The reduction of inflammatory burden, and of BNP values at 6th and 12th month of follow up has been observed in a previous study conducted on T2DM patients with HF and treated by multipolar CRTd [1, 6]. Indeed, both inflammatory markers and BNP are overexpressed in a condition of HF, and in HF patients with 

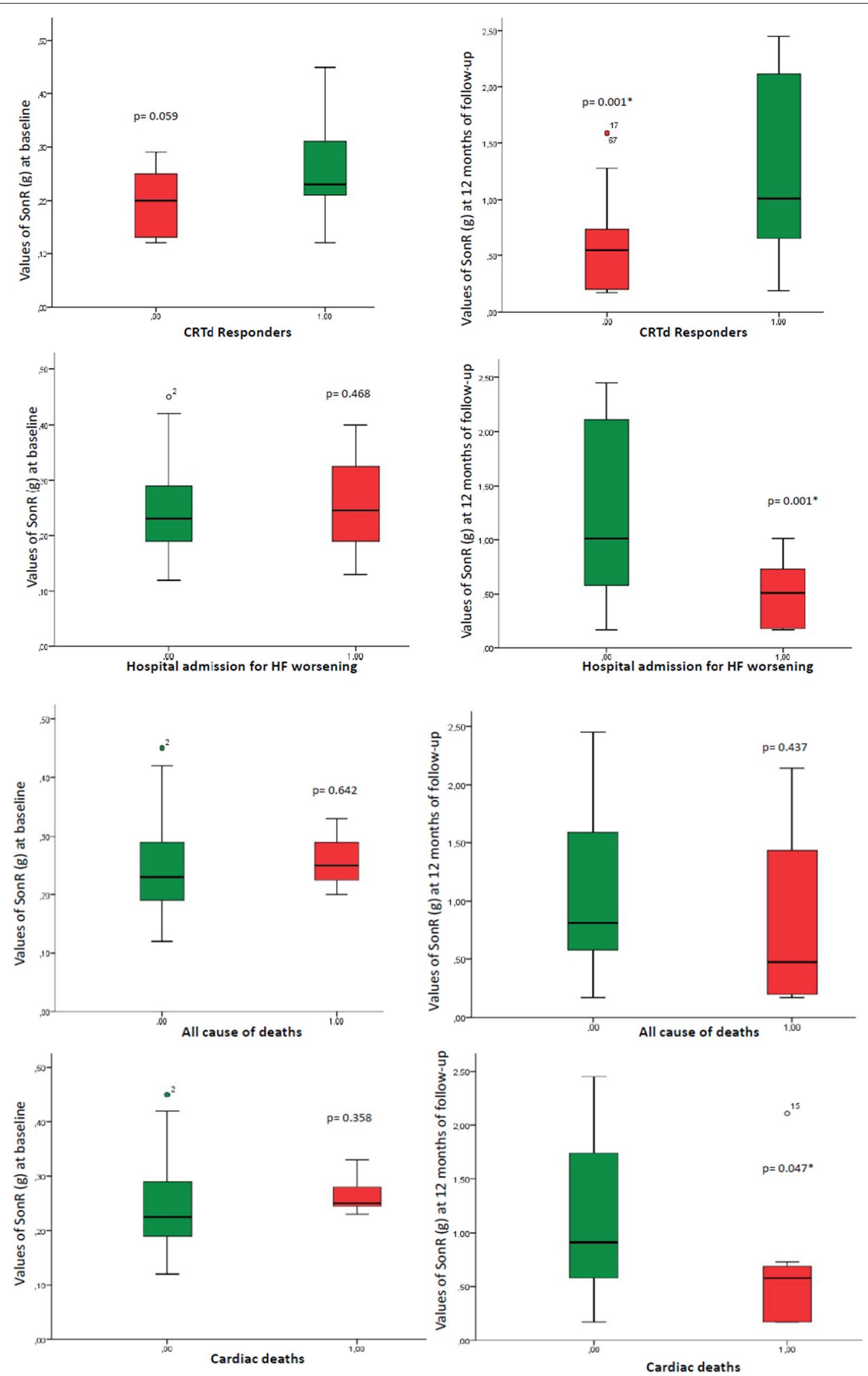
Table 3 Univariate and Multivariate Cox regression analysis for CRTd responders (a), hospitalization for HF worsening (b), cardiac deaths (c) and all cause of deaths (d)

\begin{tabular}{|c|c|c|c|c|}
\hline & Univariate analysis $\mathrm{HR}(95 \% \mathrm{Cl})$ & p value & Multivariate analysis $\mathrm{HR}(95 \% \mathrm{Cl})$ & $\mathrm{p}$ value \\
\hline \multicolumn{5}{|c|}{ A. Multivariate Cox regression analysis for parameters associated with CRT responders } \\
\hline Age & $0.102[0.11-0.968]$ & 0.048 & $0.713[0.007-1.773]$ & 0.276 \\
\hline Automatic & $0.795[0.567-1.115]$ & 0.184 & $3.636[1.271-10.399]$ & $0.016^{*}$ \\
\hline Beta blockers & $1.176[0.806-1.716]$ & 0.401 & $1.156[0.745-1.793]$ & 0.517 \\
\hline BNP & $1.001[0.989-1.101]$ & 0.868 & $1.001[0.889-1.007]$ & 0.816 \\
\hline COPD & $1.446[0.948-2.204]$ & 0.087 & $1.527[0.935-2.495]$ & 0.091 \\
\hline CRP & 1.101 [0.992-1.280] & 0.274 & 1.017 [0.995-1.041] & 0.136 \\
\hline $\mathrm{HbA1c}$ & 1.118 [0.851-1.315] & 0.643 & $1.181[0.922-1.472]$ & 0.123 \\
\hline Hypertension & 0.898 [0.619-1.302] & 0.569 & $0.895[0.561-1.430]$ & 0.644 \\
\hline LVEF & $1.006[0.970-1.044]$ & 0.736 & $1.036[0.993-1.081]$ & 0.102 \\
\hline NYHA 3 & $1.176[0.840-1.647]$ & 0.345 & $1.829[0.923-3.626]$ & 0.084 \\
\hline Obesity & 1.497 [1.290-1.852] & 0.011 & 1.330 [0.829-1.843] & 0.082 \\
\hline QRS duration & $0.989[0.971-1.008]$ & 0.255 & $0.991[0.971-1.011]$ & 0.379 \\
\hline SonR & $10.2[5.227-19.952]$ & 0.002 & $2.863[1.537-6.231]$ & $0.006^{*}$ \\
\hline $6 \mathrm{MWT}$ & 1.001 [0.993-1.007] & 0.926 & 1.010 [0.993-1.007] & 0.994 \\
\hline \multicolumn{5}{|c|}{ B. Multivariate Cox regression analysis for parameters associated with hospitalizations for heart failure } \\
\hline Age & $1.012[0.873-1.322]$ & 0.958 & $0.997[0.671-1.201]$ & 0.240 \\
\hline Automatic & $0.795[0.567-1.115]$ & 0.184 & $1.166[0.118-1.504]$ & 0.895 \\
\hline Beta blockers & $1.301[0.587-2.885]$ & 0.517 & $0.844[0.336-2.122]$ & 0.718 \\
\hline BNP & $1.002[1.001-1.301]$ & 0.011 & $1.002[1.001-1.040]$ & 0.125 \\
\hline COPD & $0.561[0.276-1.141]$ & 0.111 & $2.364[0.907-6.158]$ & 0.078 \\
\hline CRP & 1.011 [0.978-1.046] & 0.507 & 1.032 [0.982-1.084] & 0.219 \\
\hline $\mathrm{HbA1c}$ & 1.142 [0.816-1.913] & 0.143 & $0.915[0.589-1.541]$ & 0.762 \\
\hline Hypertension & 1.991 [1.003-3.952] & 0.049 & $2.503[0.809-7.745]$ & 0.111 \\
\hline LVEF & 1.029 [0.956-1.108] & 0.443 & $1.061[0.960-1.172]$ & 0.245 \\
\hline NYHA 3 & $0.531[0.258-1.096]$ & 0.087 & 0.962 [0.289-3.202] & 0.950 \\
\hline Obesity & $0.905[0.276-2.965]$ & 0.869 & $1.093[0.220-5.429]$ & 0.913 \\
\hline QRS duration & $0.964[0.927-1.020]$ & 0.066 & 0.960 [0.919-1.003] & 0.069 \\
\hline SonR & $0.074[0.004-1.292]$ & 0.074 & $0.679[0.118-1.154]$ & 0.932 \\
\hline $6 \mathrm{MWT}$ & $0.999[0.985-1.013]$ & 0.891 & $0.995[0.980-1.011]$ & 0.552 \\
\hline \multicolumn{5}{|c|}{ C. Multivariate Cox regression analysis for parameters associated with cardiac deaths } \\
\hline Age & $0.953[0.795-1.541]$ & 0.543 & $1.362[0.632-1.872]$ & 0.361 \\
\hline Automatic & $1.670[0.489-5.705]$ & 0.413 & $0.111[0.001-2.583]$ & 0.427 \\
\hline Beta blockers & $2.099[0.525-3.024]$ & 0.176 & $1.095[0.048-1.435]$ & 0.945 \\
\hline BNP & $1.001[0.998-1.004]$ & 0.681 & $0.999[0.996-1.003]$ & 0.679 \\
\hline COPD & $1.119[0.031-1.447]$ & 0.072 & $2.138[0.942-4.002]$ & 0.401 \\
\hline CRP & 0.913 [0.807-1.034] & 0.151 & $0.903[0.734-1.112]$ & 0.338 \\
\hline $\mathrm{HbA1c}$ & $1.601[0.925-2.563]$ & 0.142 & $3.224[0.841-4.389]$ & 0.106 \\
\hline Hypertension & $0.226[0.029-1.764]$ & 0.156 & $0.515[0.026-1.019]$ & 0.663 \\
\hline LVEF & $1.127[0.964-1.316]$ & 0.133 & $1.126[0.855-1.482]$ & 0.397 \\
\hline NYHA 3 & $0.615[0.180-2.102]$ & 0.439 & $0.458[0.008-2.618]$ & 0.705 \\
\hline Obesity & $2.283[0.002-3.248]$ & 0.521 & $0.759[0.001-1.621]$ & 0.993 \\
\hline QRS duration & $0.951[0.885-1.021]$ & 0.164 & $0.897[0.782-1.016]$ & 0.087 \\
\hline SonR & $0.056[0.001-8.699]$ & 0.263 & $0.010[0.001-5.267]$ & 0.520 \\
\hline $6 \mathrm{MWT}$ & $0.992[0.968-1.016]$ & 0.509 & $0.973[0.919-1.031]$ & 0.355 \\
\hline \multicolumn{5}{|c|}{ D. Multivariate Cox regression analysis for parameters associated with all cause deaths } \\
\hline Age & $1.782[1.053-2.302]$ & 0.001 & $1.362[0.809-1.780]$ & 0.563 \\
\hline Automatic & $1.471[0.570-3.795]$ & 0.425 & $1.744[0.270-2.713]$ & 0.179 \\
\hline Beta blockers & $2.009[0.582-6.490]$ & 0.270 & $3.338[0.684-6.781]$ & 0.096 \\
\hline
\end{tabular}


Table 3 (continued)

\begin{tabular}{lclll}
\hline & Univariate analysis $\mathbf{H R} \mathbf{( 9 5 \%} \mathbf{C l})$ & p value & Multivariate analysis HR (95\% Cl) & $\mathbf{p ~ v a l u e ~}$ \\
\hline BNP & $0.999[0.996-1.002]$ & 0.434 & $0.998[0.994-1.021]$ & 0.258 \\
COPD & $0.268[0.106-0.680]$ & 0.006 & $2.802[0.493-5.192]$ & 0.245 \\
CRP & $0.918[0.837-1.007]$ & 0.070 & $0.915[0.792-1.057]$ & 0.915 \\
HbA1C & $0.832[0.503-1.742]$ & 0.430 & $0.587[0.201-3.105]$ & 0.224 \\
Hypertension & $0.027[0.001-1.801]$ & 0.092 & $0.898[0.648-1.547]$ & 0.936 \\
LVEF & $1.008[0.916-1.110]$ & 0.869 & $0.879[0.001-5.644]$ & 0.713 \\
NYHA 3 & $0.292[0.096-0.888]$ & 0.030 & $1.966[0.030-3.082]$ & 0.465 \\
Obesity & $2.289[0.014-3.766]$ & 0.407 & $0.468[0.009-1.821]$ & 0.099 \\
QRS duration & $0.958[0.907-1.012]$ & 0.128 & $0.958[0.885-1.037]$ & 0.284 \\
SonR & $0.191[0.005-7.540]$ & 0.378 & $1.684[0.101-5.647]$ & 0.713 \\
6MWT & $1.009[0.991-1.028]$ & 0.339 & $1.004[0.977-1.031]$ & 0.791 \\
\hline
\end{tabular}

Italic values indicate a significant $p$ value $(p<0.05)$

* Is for statistical significant ( $p<0.05$ ). BNP, B type natriuretic peptide; COPD, chronic obstructive pulmonary disease; CRP, $C$ reactive protein; HbA1c, glycated hemoglobin 1Ac type; LVEF, left ventricle ejection fraction; NYHA 3, New York Heart Association 3 class; SonR, values of SonR signals; 6 MWT, 6 min walking test. Multivariate Cox regression analysis for parameters associated with CRT responders

depressed cardiac pump [1, 6]. In this context, BNP is a valuable marker of $\mathrm{HF}$, and a predictor of hospitalizations for HF worsening and of worse prognosis in CRTd patients $[1,6,13]$. However, BNP could be relapsed in condition of stable and unstable HF, and used for risk stratification in patients with acute and chronic HF [14]. Therefore, BNP is an independent marker of worse prognosis for patients with the failure of cardiac pump [14], and in those treated with CRTd $[1,6,13,15,16]$. Consequently, T2DM patients with severe reduction of cardiac pump, as evidenced by lowest values of LVEF at echocardiography, could experience a worse clinical prognosis $[1,6,15,16]$. To date, the cardiac pump reduction, in HF patients with T2DM treated with CRTd, could be caused by advanced anatomical degree of ventricular remodeling [15-20], and reflected by the loss of heart synchronism during diastolic and systolic cardiac phases $[8,10]$. In this setting, the alterations of AV/IV intervals are linked to, and could mark CRTd patients that evidenced the loss of cardiac synchronism [20]. Indeed, the CRTd patients with highest AV/IV delays could experience worse prognosis by the loss of AV and IV synchronism, and by the worsening of cardiac pump [20]. Therefore, the increase of cardiac pump could lead to the amelioration of clinical outcomes in T2DM patients with CRTd, such as previously observed in overall population of CRTd patients [10]. In addition, we reported an increase of LVEF, with reverse remodeling, and amelioration of NYHA class and clinical status in T2DM patients, which evidenced at baseline highest SonR values, that could be seen as index of best AV/IV synchronism. Thus, we could report that a best optimization of AV/IV delays could lead to best clinical outcomes for CRTd patients [8, 10]. Moreover, in our study we observed and confirmed these results in a selective population of T2DM patients with CRTd. In addition, for first time in literature, we monitored the modification of SonR values for 12 months of follow-up in diabetics with CRTd regards CRTd responders rate, hospitalizations for HF worsening, cardiac deaths and all cause of deaths. Thus, we might speculate that, the automatic vs. echo-guided approach for optimization of CRTd, could be superior to achieve CRTd responders target, and to reduce hospitalizations for HF worsening in patients with T2DM. However, we could summarize the most important functions of automatic CRTd as monitor and activator of cardiac remodeling processes, that are involved in clinical prognosis of CRTd patients. Therefore, it could be relevant to identify at baseline T2DM patients with highest values of $\mathrm{dP} / \mathrm{dT}$ signals. Indeed, these patients could have lowest AV/IV delays and best cardiac synchronism [18]. Furthermore, it looks intuitive to say that these patients could have a higher possibility to become CRTd responders, and to experience a best clinical prognosis. This point is relevant, because it opens a new scenario in the possibility to identify and to treat at best we can CRTd patients with T2DM at different stages of cardiac dyssynchrony. However, we might speculate to choice specific treatments, that in addition to automatic optimization of AV/IV delays could result in best clinical response in CRTd patients with T2DM. Finally, this could be used to ameliorate CRTd responders and to reduce worse prognosis in failing heart patients with T2DM. 


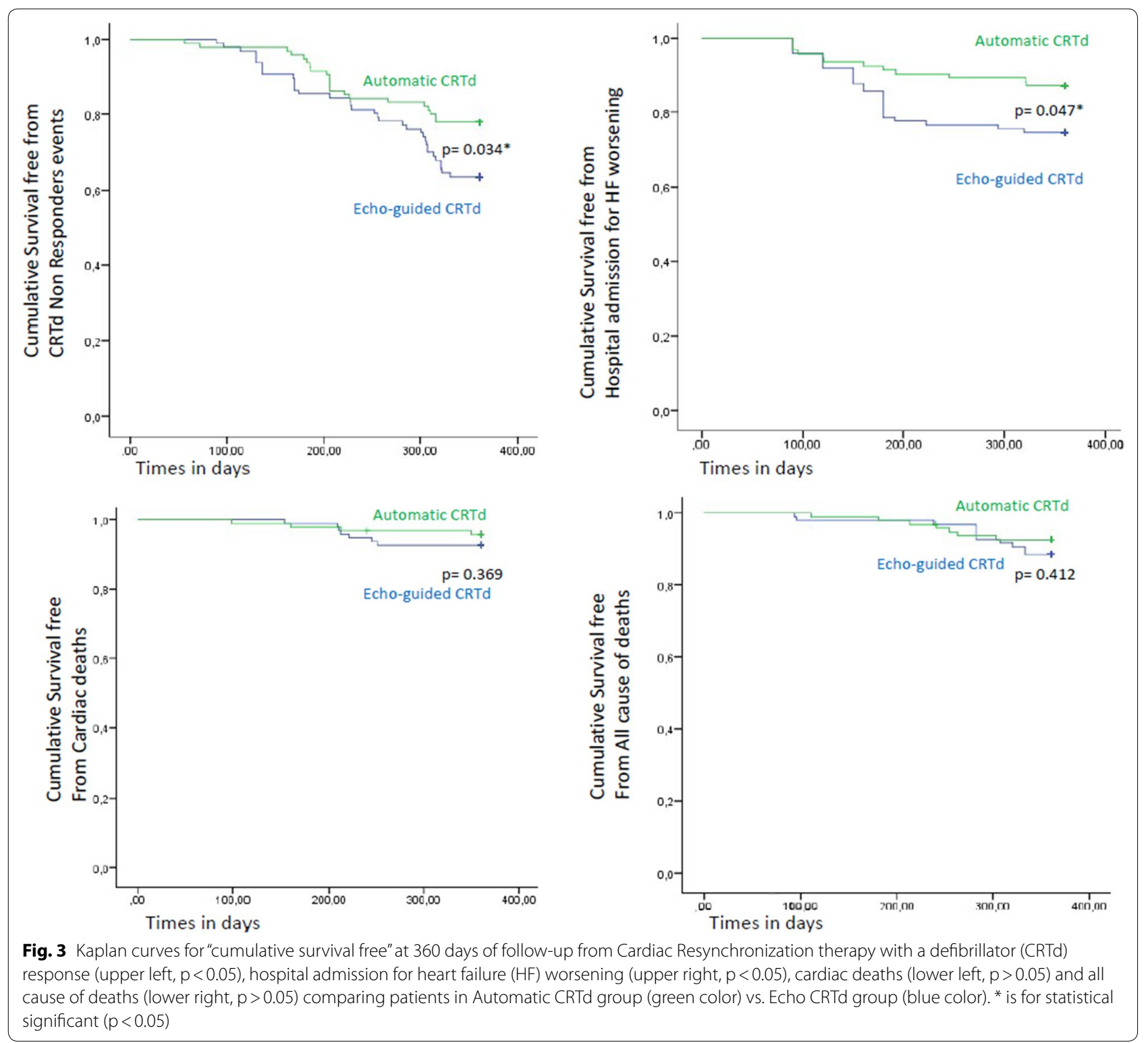

\section{Study limitations}

This study has few limitations. As first, the small sample size and the duration of follow-up could influence study results, that have to be applied in a future study with larger size of T2DM patients, and at more long term follow up analysis. In addition, in the present study by the loss of an experimental animal model of HF with automatic vs. echo guided CRTd, we did not practice cardiac biopsy to show the different inflammation/fibrosis for the main study outcomes. In addition, we did not use a continuous monitoring systems for arrhythmias detection and devices interventions as described by authors [21], and this may affect the study outcomes. Therefore, further studies are needed to better understand the pleiotropic functions of automatic CRTd guided by SonR, and its cardiovascular effects in terms of AV/IV synchronism and best clinical outcomes. Therefore, a larger clinical trial may be adequate to assess all these pathogenic processes in a population of failing heart patients with T2DM treated by automatic CRTd. This may be applied in clinical practice to reduce hospitalizations, and to improve CRTd response in failing heart patients with T2DM.

\section{Conclusions}

Our study results evidenced that automatic vs. echo guided CRTd optimization increased significantly the CRTd responders rate, and reduced hospitalizations 
for HF worsening in T2DM patients. To date, SonR signals showed a significant modification regards CRTd responders, hospitalizations for HF worsening events, and cardiac deaths. Notably and clinically relevant, the automatic optimization of AV/IV delays could increase of more than 3 folds the possibility to become CRTd responders, and baseline highest values of SonR signals could characterize patients with 2.8 folds higher possibility to become CRTd responders. Therefore, we could suggest to opt for SonaR guided CRTd implant, to reach the best cardiac synchronism, and to increase the possibility for a T2DM patient to become CRTd responder and to reach best clinical prognosis.

\section{Supplementary information}

Supplementary information accompanies this paper at https://doi. org/10.1186/s12933-020-01180-8.

Additional file 1: Table S1. Clinical characteristics of study population at 6th month of follow-up in overall, and automatic vs. echo-guided patients. BMI: body mass index; BNP: B type natriuretic peptide; CRP: C reactive protein; HbA1c: glycated hemoglobin 1Ac type; IL-6: interleukine 6: LVEDd: left ventricle end diastolic diameter; LVEDv: left ventricle end diastolic volume; LVEF: left ventricle ejection fraction; LVESd: left ventricle end systolic diameter; LVESv: left ventricle end systolic volume; NYHA II, III: New York Heart Association II and III class; SonR: values of SonR signals; TNFa: tumor necrosis factor alpha; 6MWT: 6 minutes walking test. ${ }^{* *}$ is for statistical significant $(p<0.05)$.

\section{Abbreviations}

AV: Atrioventricular; BNP: B type natriuretic peptide; CRP: C reactive protein: CRTd: Cardiac resynchronization therapy with defibrillator; HbA1c: Glycated hemoglobin 1Ac type; HF: Heart failure; ICD: Implantable cardioverter defibrillator; IEGM: Intracardiac electrogram; IL6: Interleukin-6; IV: Interventricular; LV: Left-ventricular; LVEDD: Left-ventricular end-diastolic diameter; LVEDV: Leftventricular end-diastolic volume; LVEF: Left ventricle ejection fraction; LVESD: Left-ventricular end-systolic diameter; LVESV: Left-ventricular end-systolic volume; NYHA: New York Heart Association; TNF a: Tumor necrosis factor-a: T2DM: Type 2 diabetes mellitus; VTI:Velocity-time integral; 6MWT: Six minutes walking test.

\section{Acknowledgments}

All authors have equally contributed to this research. This research did not receive any specific grant from funding agencies in the public, commercial, or not-for-profit sectors.

\section{Authors' contributions}

CS: wrote the research project and the full manuscript. PP: data collection and interpretation; CS, MS, AR, and VD: performed CRTd implants; GP, RM, MM: study revision editing, and data analysis; RM: manuscript editing. All authors read and approved the final manuscript.

\section{Funding}

None to declare.

\section{Availability of data and materials \\ Data and study materials are available.}

\section{Ethics approval and consent to participate}

Ethical Committee of University of Campania "Luigi Vanvitelli", Catholic University of Sacred Heart, Gemelli Molise and Vecchio Pellegrini Hospital approved the research protocol and gave the consent to participate in the study.

\section{Consent for publication}

Authors give the full consent to publish the present article.

\section{Competing interests}

C.S edited and wrote the research project and the full manuscript.

\section{Author details}

${ }^{1}$ Department of Advanced Medical and Surgical Sciences, University of Campania "Luigi Vanvitelli", Piazza Miraglia 2, 80131 Naples, Italy. ${ }^{2}$ Unit of Cardiology, Department of Experimental, Diagnostic and Specialty Medicine-DIMES, University of Bologna, Bologna, Italy. ${ }^{3}$ Unit of Cardiovascular Diseases and Arrhythmias, "Vecchio Pellegrini" Hospital, Naples, Italy. ${ }^{4}$ Unit of Cardiovascular Diseases and Arrhythmias, "Gemelli Molise", Campobasso, Italy. ${ }^{5}$ Department of Cardiac Surgery and Cardiovascular Diseases, "Catholic University of Sacred Heart", Rome, Italy. ${ }^{6}$ Unit of Cardiovascular Diseases and Arrhythmias, "Antonio Cardarelli" Hospital, Naples, Italy.

Received: 6 October 2020 Accepted: 15 November 2020

Published online: 28 November 2020

\section{References}

1. Sardu C, Barbieri M, Santamaria M, et al. Multipolar pacing by cardiac resynchronization therapy with a defibrillators treatment in type 2 diabetes mellitus failing heart patients: impact on responders rate, and clinical outcomes. Cardiovasc Diabetol. 2017;16(1):75.

2. Cleland JG, Daubert JC, Erdmann E, et al. Cardiac ResynchronizationHeart Failure (CARE-HF) Study Investigators. The effect of cardiac resynchronization on morbidity and mortality in heart failure. N Engl J Med. 2005:352(15):1539-49.

3. Auricchio A, Prinzen FW. Non-responders to cardiac resynchronization therapy: the magnitude of the problem and the issues. Circ J. 2011;75:521-7.

4. Daubert C, Behar N, Raphael PM, Mabo P, Leclerca C. Avoiding nonresponders to cardiac resynchronization therapy: a practical guide. Eur Heart J. 2017;38:1463-72.

5. Cuoco FA, Gold MR. Optimization of cardiac resynchronization therapy: importance of programmed parameters. J Cardiovasc Electrophysiol. 2012;23:110-8.

6. Sardu C, Paolisso P, Sacra C, et al. Cardiac resynchronization therapy with a defibrillator (CRTd) in failing heart patients with type 2 diabetes mellitus and treated by glucagon-like peptide 1 receptor agonists (GLP-1 RA) therapy vs. conventional hypoglycemic drugs: arrhythmic burden, hospitalizations for heart failure, and CRTd responders rate. CardiovasC Diabetol. 2018;17(1):137.

7. Niazi I, Baker J, Corbisiero R, et al. MPP Investigators. Safety and efficacy of multipoint pacing in cardiac resynchronization therapy: the multipoint pacing trial. JACC Clin Electrophysiol. 2017;3(13):1510-8.

8. Ritter P, Delnoy PPH, Padeletti L, et al. A randomized pilot study of optimization of cardiac resynchronization therapy in sinus rhythm patients using a peak endocardial acceleration sensor vs standard methods. Europace. 2012a;14:1324-33.

9. Kamdar R, Frain E, Warburton F, et al. A prospective comparison of echocardiography and device algorithms for atrioventricular and interventricular interval optimization in cardiac resynchronization therapy. EP Europace. 2009;12:84-91.

10. Brugada J, Delnoy PD, Brachmann J, et al. for the RESPOND CRT Investigators. Contractility sensor-guided optimization of cardiac resynchronization therapy: results from the RESPOND-CRT trial. European Heart J. 2017:38:730-8.

11. Moghissi ES, Korytkowski MT, DiNardo M, et al. American Association of Clinical Endocrinologists; American Diabetes Association. American Association of Clinical Endocrinologists and American Diabetes Association consensus statement on inpatient glycemic control. Diabetes Care 2009;32(6):1119-31.

12. Yancy CW, Jessup M, Bozkurt B, et al. 2017 ACC/AHA/HFSA focused update of the 2013 ACCF/AHA guideline for the management of heart failure: a report of the American College of Cardiology/American Heart Association Task Force on Clinical Practice Guidelines and the Heart Failure Society of America. J Am CollCardiol. 2017;70(6):776-803. 
13. Sardu C, Santamaria M, Funaro S, Sacra C, Barbieri M, Paolisso P, Marfella R, Paolisso G, Rizzo MR. Cardiac electrophysiological alterations and clinical response in cardiac resynchronization therapy with a defibrillator treated patients affected by metabolic syndrome. Medicine (Baltimore). 2017;96(14):e6558.

14. Ritter P, Delnoy PPH, Padeletti L, et al. A randomized pilot study of optimization of cardiac resynchronization therapy in sinus rhythm patients using a peak endocardial acceleration sensor vs. standard methods. Europace. 2012b;14:1324-33

15. Gorcsan J III, Abraham T, Agler DA, et al. Echocardiography for cardiac resynchronization therapy: recommendations for performance and reporting - a report from the American Society of Echocardiography Dyssynchrony Writing Group endorsed by the Heart Rhythm Society. J Am Soc Echocardiogr. 2008;21:191-213.

16. Kerlan JE, Sawhney NS, Waggoner AD, et al. Prospective comparison of echocardiographic atrioventricular delay optimization methods for cardiac resynchronization therapy. Heart Rhythm. 2006a;3:148-54.

17. Petretta M, Colao A, Sardu C, et al. NT-proBNP, IGF-I and survival in patients with chronic heart failure. Growth Horm IGF Res. 2007;17(4):288-96.
18. Sardu C, Barbieri M, Rizzo MR, Paolisso P, Paolisso G, Marfella R. Cardiac resynchronization therapy outcomes in Type 2 diabetic patients: role of MicroRNA changes. J Diabetes Res. 2016;2016:7292564.

19. Marfella R, Di Filippo C, Potenza N, et al. Circulating microRNA changes in heart failure patients treated with cardiac resynchronization therapy: responders vs. non-responders. Eur J Heart Fail. 2013;15(11):1277-88.

20. Sardu C, Marfella R, Santulli G, Paolisso G. Functional role of miRNA in cardiac resynchronization therapy. Pharmacogenomics. 2014;15(8):1159-68.

21. Sardu C, Santamaria M, Rizzo MR, et al. Telemonitoring in heart failure patients treated by cardiac resynchronisation therapy with defibrillator (CRT-D): the TELECART Study. Int J Clin Pract. 2016;70(7):569-76.

\section{Publisher's Note}

Springer Nature remains neutral with regard to jurisdictional claims in published maps and institutional affiliations.
Ready to submit your research? Choose BMC and benefit from:

- fast, convenient online submission

- thorough peer review by experienced researchers in your field

- rapid publication on acceptance

- support for research data, including large and complex data types

- gold Open Access which fosters wider collaboration and increased citations

- maximum visibility for your research: over $100 \mathrm{M}$ website views per year

At BMC, research is always in progress.

Learn more biomedcentral.com/submissions 